\title{
Présentation du numéro
}

\section{Jalons pour une anthropologie clinique}

Le monde de la psychiatrie et de la psychologie clinique d'aujourd'hui semble de plus en plus se caractériser par un morcellement en diverses spécialisations, s'affirmant davantage dans une rivalité les unes aux autres que dans une complémentarité au service de l'être humain souffrant. Chaque orientation allant de ses principes théoriques et défendant son étiopathologie concourt à l'éclatement de la pratique en une multitude de facettes irréductibles et exclusives. Une telle tendance risque du même coup de porter préjudice au malade luimême, écartelé entre les différentes visions de son profil psychopathologique. Si la psychiatrie et la psychologie clinique souffrent d'un manque d'intégration, il convient donc de réfléchir à ce qui pourrait fédérer leurs diverses orientations: est-il possible de les concevoir de manière plus concertante, au-delà du modèle bio-psycho-social ou d'une approche multifactorielle, qui en général ne soulignent pas suffisamment les points d'articulation existant entre les différents domaines étudiés ? En effet, plutôt que de s'arrêter aux seuls discours étiopathologiques qui souvent s'excluent, considérons davantage les pathogenèses qui, elles, peuvent se composer, se succéder, "se commenter réciproquement », selon le mot de Weizsäcker.

Repenser la clinique dans une visée plus intégrative mais critique, en évitant le réductionnisme fragmentaire qui la menace, tel est justement le propos de l'anthropologie clinique. Pour celle-ci, la défense d'une pluralité de modèles psychopathologiques et psychothérapeutiques impose qu'on s'interroge sur leurs fondements historiques, philosophiques et épistémologiques, dans une démarche où l'être humain comme patient est saisi dans sa dimension tout à la fois subjective et intersubjective. Clinique du sujet, d'inspiration humaniste, se référant explicitement à l'anthropologie phénoménologique, avec les apports d'une anthropologie aussi bien sociale et culturelle que politique, l'anthropologie clinique propose donc le détour par les sciences humaines (histoire, philosophie, linguistique, sociologie, éthique, etc.) pour mieux revenir à l'être subjectif et en situation du patient. Celuici est appréhendé dans son expérience vécue d'homme souffrant avant de l'être dans ses fonctions.

C'est dans un tel projet d'anthropologie clinique que Nicolas Duruz, professeur de psychologie clinique et Raphaël Célis, professeur de philosophie, ont co-organisé à l'Université de Lausanne en novembre 2005, avec l'aide soutenue d'Hervé Mésot et de Franca Madioni, un colloque international intitulé «Psychopathologie et psychothérapie au regard de l'anthropologie clinique ». Différents spécialistes reconnus, provenant de diverses orientations, y ont apporté leur contribution. Ce colloque a su offrir un espace de dialogue et de réflexion aux divers représentants de la clinique (psychiatrie, psychanalyse, systémique, neurosciences, sciences cognitives...), ainsi qu'aux théoriciens, penseurs et philosophes présents. Son succès a motivé les organisateurs à faire appel à d'autres praticiens et chercheurs qui n'avaient pu s'exprimer à cette occasion afin de constituer un recueil d'articles élargissant les actes du colloque.

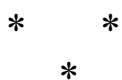


Le recueil d'articles s'ouvre avec trois contributions voulant donner un cadre à ce qui est dénommé Anthropologie clinique, appellation à ce jour encore non contrôlée! Dans une introduction à l'anthropologie clinique dont il a soin de laisser ouverts les contours, Hervé MESOT rappelle dans ses grandes lignes la pensée de Ludwig Binswanger qui, en tant que fondateur de la psychiatrie phénoménologique, constitue une des sources principales de cette anthropologie. Cette présentation est précédée d'une brève histoire des idées et des concepts qui ont donné le jour à l'anthropologie clinique. Disciple de Binswanger, comme d'autres grands maîtres d'ailleurs, Jacques SCHOTTE prolonge cette introduction en rappelant quelques principes théoriques essentiels de son " anthropopsychiatrie » : via l'héritage fondamental de Szondi, il opère une synthèse magistrale entre la théorie freudienne des pulsions et la conception de l'homme comme existant. C'est dans la méthode « pathoanalytique » qu'il s'est employé à promouvoir comme méthode d'analyse de la condition humaine à partir des comportements psychopathologiques, que réside le point d'orgue de sa démarche, à la faveur de laquelle la psychiatrie est élevée au rang de médecine tout à la fois scientifique et irréductiblement humaine. Davantage centrée sur le monde des psychothérapies, dont la pluralité des méthodes n'est pas sans effet délétère sur les patients, le tiers public et les psychothérapeutes eux-mêmes, la contribution de Nicolas DURUZ présente l'anthropologie clinique comme un carrefour possible de réflexion théorique et clinique : un dialogue est-il possible entre ces méthodes, sans que l'échange n'aboutisse pour autant à une sorte d'esperanto psychothérapeutique ? A la faveur de la réduction phénoménologique et donc d'un travail de clarification épistémologique, tout psychothérapeute est invité à prendre conscience de la portée et de la limite de l'approche à laquelle il prête obédience.

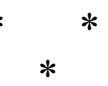

Dès le départ, la phénoménologie clinique s'est nourrie de la pensée psychanalytique ; leur rencontre a souvent été très féconde, à l'image de celle qui eut lieu entre leurs fondateurs, Freud et Binswanger, toujours restée amicale même dans leurs divergences. L'anthropologie clinique s'alimente grandement de cet échange, comme le mentionne déjà le travail de Schotte. Les six contributions suivantes en témoignent encore, chacune à leur manière. Henri MALDINEY décrit toute la fécondité de sa méthode sur un terrain où Freud excellait déjà celui de l'analyse des rêves - mais en soulignant le pas gagné : celui de la compréhension de cette forme d'existence à l'impossible qu'est la psychose, et pour laquelle Freud ne disposait encore que de quelques pistes. Dans son article «L'intersubjectif et l'intrasubjectif dans l'expérience de souffrance. Un regard psychanalytique », Monique SCHNEIDER part aussi du monde du rêve, tel que Freud l'analyse. Elle montre comment le rêveur tend à éjecter vers autrui une de ses «personnes psychiques »; il s'agit bien souvent de l'enfant de souffrance qu'il porte en lui. Elle y repère alors la création d'un espace intersubjectif appelé à hériter des scissions qui sont à la source de l'espace intrasubjectif. Ce qui lui permet, après une démarche de pensée rigoureuse, d'éclairer très finement la position intersubjective qui sous-tend le travail analytique : une tentative de rencontre entre la détresse de l'analysant et la présence de l'analyste qui, à la faveur de son attention flottante, pourra accueillir cette détresse et permettre à l'analysant de se la réapproprier dans son propre espace intrasubjectif. Caroline Gros, quant à elle, met au jour la dimension phorique (Tragung) et thymique du transfert (Übertragung), faisant ainsi apparaître l'un des apports majeurs de Binswanger dans la construction de l'espace analytique d'inspiration freudienne. Franca MADIONI montre aussi que Binswanger n'a cessé de retravailler la notion freudienne de transfert, et que ses réticences à l'égard de l' " homo natura », qu'il reprochait à Freud de privilégier à l'excès, ne l'ont pas empêché de reprendre à son compte des hypothèses psychanalytiques tout à fait 
centrales. L'article de Jean NAUDIN et son équipe vise à expliciter le rôle joué par la réduction phénoménologique dans le traitement thérapeutique du patient conçu comme un cas singulier. Si la psychiatrie construit en effet son diagnostic sur la base d'une typologie nosographique, c'est néanmoins l'attention portée à l'événementiel, et donc à ce qui individualise radicalement 1' «essence» ou le «type» psychopathologique prédéfini, qui permet d'instaurer un nouveau cadre de raisonnement où l'emporte l'histoire intérieure du patient. Enfin, dans son exposé sur la psychothérapie des cas difficiles, Mareike WoLF-FEDIDA fait le point sur l'évolution de l'anthropologie clinique depuis Sigmund Freud et Ludwig Binswanger jusqu'à l'anthropopsychiatrie de Jacques Schotte. Elle fait ressortir en quoi le modèle anthropologique la fait s'éloigner d'une psychiatrie exclusivement prescriptive où l'écoute du patient n'a pour fonction que d'informer le diagnostic sans que ne lui soit accordée aucune portée thérapeutique. Elle démontre ensuite l'efficacité de sa démarche dans le traitement d'un patient psychotique âgé pour lequel, a priori, l'entreprise d'une psychothérapie aurait pu apparaître comme destinée à l'échec.

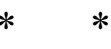

Roland Kuhn a déclaré un jour que l'on ne peut mesurer jusqu'à quel degré le psychisme s'enracine dans l'organisme et inversement. Husserl, le père de la phénoménologie, et Merleau-Ponty, un de ses éminents représentants en France, ont exprimé une intuition semblable lorsqu'ils ont insisté sur la nécessité de mener les recherches en biologie dans deux directions conjointes : la première, naturaliste, est celle qui se pratique le plus communément. La seconde, dont l'origine remonte au moins jusqu'à Goethe, et dont le plus éminent représentant est aujourd'hui André Pichot, réintroduit le sujet à même l'étude du vivant humain et procède à une analyse de son interaction avec le milieu qui s'étend des perceptions et des kinesthèses, pour se prolonger ensuite dans des activités plus complexes. Pierre BOVET, Françoise SCHENK et Luca COCCHI, Frédéric MAURIAC et Nathalie DEPRAZ empruntent d'une manière ou d'une autre une voie analogue. Pierre BOVET présente une hypothèse neurodéveloppementale partiellement explicative de la genèse des troubles du spectre schizophrénique, qui l'aide à mieux dégager phénoménologiquement, en réhabilitant la notion psychopathologique de schizoïdie, ce qu'il pourrait y avoir de spécifique au mode d'existence de ces sujets, et cela au-delà d'une simple énumération des symptômes. Dans leur contribution Françoise SCHENK et Luca COCCHI cherchent à mettre en évidence des analogies de structures entre les différents niveaux du vivant et du mental, en décrivant par exemple les mécanismes complémentaires cérébraux qui rendent possible, dans l'acte de cognition, la combinaison du « voir pour faire » et du « voir pour nommer ». Quant à Frédéric MAURIAC et Nathalie DEPRAZ », dans un texte riche et condensé, ils proposent de dépasser une conception de la relation thérapeutique comme mise en présence de deux individus (à la première et à la troisième personne) au profit d'une relation dynamique entre deux sujets, qui se décline sur le mode de la seconde personne. Pour le dire succinctement, on pourrait suggérer que tous ces auteurs tentent d'opérer un chiasme entre deux types de réduction. La première fait l'économie des contenus de sens intentionnels ou inconscients, pour se concentrer exclusivement sur les processus neurophysiologiques; la seconde met ces derniers entre parenthèses pour s'attacher à la description de la relation que le sujet entretient avec le monde et avec lui-même. Au terme de cette double opération, ce sont bien entendu les corrélations susceptibles d'être articulées qui importent, car elles permettent de soutenir les entretiens psychothérapeutiques par une attitude scientifique adéquate. 
Si l'on admet qu'un sujet sans société est aussi absurde qu'un sujet sans corps - un autre postulat de l'anthropologie clinique selon laquelle l'étude de l'individu dans ses dimensions bio-psycho-sociales s'avère insuffisante quand elle fait l'économie de l'expérience subjective et intersubjective de cet individu -, on comprendra pourquoi ce volume réunit plusieurs contributions qui ont en commun d'inscrire l'expérience de la souffrance psychique, l'acte de la nommer (diagnostic) et de la traiter (thérapie), dans le contexte social où elle se déploie. Le " social » y est donc convoqué selon ses différentes configurations, non comme une variable supplémentaire, mais comme ce qui détermine intrinsèquement l'être-au-monde.

C'est donc tout naturellement que l'éthique, en tant que science du rapport des actions humaines avec les valeurs qui les gouvernent, se trouve au cœur de la contribution de Muriel GILBERT. Celle-ci reprend la notion d'identité narrative de Ricœur pour l'intégrer dans une anthropologie du sujet souffrant, marqué par l'impuissance du pâtir, en train de faire le récit de sa vie propre. Dans son texte « Nommer la souffrance pour soi et pour l'autre : un regard dialogique », Michèle Grossen, psychosociologue, s'interroge à travers trois situations cliniques sur les conditions « dialogiques » de la rencontre thérapeutique : la souffrance n'est jamais un phénomène à caractère strictement privé ; elle s'exprime et se nomme dans le langage qui est toujours déjà un produit intersubjectif, un espace public, lourd de passé et d'ailleurs, d'altérité et d'appartenances. Ce qui nous conduit tout naturellement à la prochaine contribution. Elle vise la dimension politique du « social », à l'œuvre dans l'anthropogenèse des individus. L'anthropologie clinique n'y est pas étrangère non plus. Si de ce point de vue une contribution directement inspirée par les recherches transculturelles et interculturelles eût été bienvenue - ces recherches sont toujours utiles pour nous aider à penser la différence, sacrifiée souvent à l' " évidence » de l'universel -, nous pouvons heureusement compter sur la contribution novatrice de Jean-Marie LACROSSE. Elle montre comment dans nos sociétés postmodernes, soumises à une individualisation forcenée, l'accès au statut de personne est problématique pour les individus : s'activant comme autant de particules non reliées au tout, ils n'ont plus la capacité de se regarder et d'agir du point de vue de l'ensemble politique ; ils ne peuvent être que livrés à une quête incessante d'autonomie. L'auteur cherche alors à rendre compte de nouvelles figures de la pathologie mentale, où l'on retrouve à chaque fois la bipolarité de comportements d'indépendance et de dépendance exacerbés.

Les deux dernières contributions nous ramènent à une lecture directement phénoménologique de la pathologie, tout en restant cependant sensibles aux fragilités du tissu social dont souffrent nos sociétés. Consacrée à une étude phénoménologique de la dépression, la contribution fouillée de Raphaël CELIS emprunte en quelque sorte la démarche de Freud dans "Malaise dans la Civilisation ». Ce qui s'avère plus actuel, c'est la nature même des facteurs pathogènes à la faveur desquels les dépressions, devenues endémiques, sont aujourd'hui déclenchées : les crispations identitaires causées entre autres par l'effondrement du modèle patriarcal, la concurrence sans merci que se livrent les individus au nom du néolibéralisme, l'absence d'idéaux collectifs, les migrations massives, la déculturation des élites et bien d'autres phénomènes encore - ce qui n'est pas sans rejoindre sur certains points l'analyse précédente de Lacrosse. Le bel article, fougueux et chaleureux, de Graziano MARTIGNONI, "Fragments d'anthropologie clinique en action », qui se défie d'un monde d'hyper-transparence où le négatif est occulté, dénonce une psychiatrie qui ne parvient plus à considérer des individus en souffrance tels qu'ils se présentent en "chair et en os », mais seulement des entités nosographiques abstraites. Il nous fait prendre la mesure des séismes collectifs auxquels les individus les plus fragiles sont sommés de s'adapter, non sans dommages. En raison peut-être de l'attention qu'elle a toujours portée depuis Husserl au 
« monde de la vie », la phénoménologie clinique s'avère particulièrement lucide sur ce point : les pathologies psychiques ne peuvent le plus souvent éclore que parce qu'elles constituent des réponses à un environnement qui les nourrit.

Nous osons espérer que les contributions de ce recueil, présentées et rédigées par l'équipe lausannoise, avec l'apport de chercheurs collaborant depuis de nombreuses années avec elle, jouiront d'une large audience auprès des spécialistes de la maladie mentale. Promouvoir l'anthropologie clinique comme lieu de pensée et d'échanges au service de l'humanité du patient, tel est bien en fin de compte le projet du collectif lausannois.

\section{N. Duruz, ${ }^{1}$ R. Célis, ${ }^{2}$ V. Dallèves ${ }^{3}$}

Lausanne, le 25 janvier 2007

\footnotetext{
${ }^{1}$ Professeur de Psychologie clinique à l'Université de Lausanne. Codirecteur de l'Institut Universitaire de Psychothérapie au Département de Psychiatrie - CHUV

${ }^{2}$ Professeur de Philosophie à l'Université de Lausanne

${ }^{3}$ Licencié en Philosophie. Etudiant en Master de Psychologie. Coordinateur du projet d'édition
} 